\title{
RISCO E VULNERABILIDADE SOCIAL A DESASTRES NATURAIS NO BRASIL: PROPOSTA DE UM ARCABOUÇO PARA INDICADORES MULTIESCALARES
}

\author{
Gustavo Henrique Naves Givisiez ${ }^{(a)}$, Elzira Lúcia de Oliveira ${ }^{(b)}$,
}

(a) Professor do Departamento de Geografia de Campos, UFF, gh_naves@id.uff.br

(b) Professora do Departamento de Geografia de Campos, UFF, elziralucia@id.uff.br

\section{Eixo: Geografia Física e Desastres Naturais}

\begin{abstract}
Resumo
Todos os anos, os desastres naturais resultam numerosos mortos e feridos e em expressivas perdas econômicas. O cenário de mudanças climáticas que se apresenta ao mundo é ainda um fator agravante para os eventos climáticos que podem apresentar características mais virulentas e destrutivas ao longo deste e dos próximos séculos. As consequências dos desastres naturais são ainda sentidas de forma desigual entre as pessoas: a população menos favorecida, crianças e idosos são frequentemente os mais afetados pelos desastres naturais em todo o planeta. A proposta deste artigo é apresentar uma revisão sobre um conjunto de métodos e técnicas de mensuração da vulnerabilidade a desastres naturais, e propor um arcabouço preliminar que servirá de base para a construção de um indicador de Risco a Desastres Naturais, que seja aplicável (ou ajustável) a múltiplas escalas e coerente com as bases de dados disponíveis e com os desastres mais comuns no Brasil.
\end{abstract}

Palavras chave: Desastres Naturais; Indicadores; Vulnerabilidade Socioambiental

\section{Introdução}

Todos os anos, os desastres naturais resultam numerosos mortos e feridos e em expressivas perdas econômicas. O cenário de mudanças climáticas que se apresenta ao mundo é ainda um fator agravante para os eventos climáticos que podem apresentar características mais virulentas e destrutivas ao longo deste e dos próximos séculos.

A tabela 1 resume a frequências de desastres por tipo e subtipo, total de óbitos e total de pessoas atingidas por desastres naturais (feridos, desabrigados e afetados), no mundo, entre 1970 e 2016, com base em dados do EM-DAT ${ }^{1}$. Nota-se que embora os terremotos sejam os responsáveis pelo maior número de óbitos nos últimos 46 anos, o total de afetados pelas secas e enchentes apresentam quantidades superiores a 2,5 bilhões de pessoas em valores acumulados. Em média, as secas, nos últimos 46 anos, têm afetado aproximadamente 4 milhões de pessoas evento registrado, e as enchentes fluviais tem afetado próximo a um milhão de pessoas por enchente.

1 O EM-DAT é mantido pelo Centre for Research on the Epidemiology of Disasters CRED (disponível em http://www.emdat.be) e contem mais de 12 mil registros de desastres de 1900 até o presente, incluindo número de óbitos, pessoas afetadas e perdas econômicas. Baseia-se em registros na imprensa de língua francesa e inglesa. 
XVII Simpósio Brasileiro

de Geografia Fisica Aplicada

I Congresso Nacional de Geografia Física

\section{OS DESAFIOS DA GEOGRAFIA FISICA NA FRONTEIRA DO CONHECIMENTO Instituto de Geociências - Unicamp Campinas - SP \\ 28 de Junho à 02 de Julho de 2017}

Tabela 1

Total de Desastres Naturais, Óbitos e Pessoas Afetadas por Tipo e Subtipo de Desastre. Mundo. 1970-2016

\begin{tabular}{|c|c|c|c|c|c|c|}
\hline \multicolumn{2}{|c|}{ Desastre Natural } & \multirow{2}{*}{$\begin{array}{c}\text { Freq. } \\
\text { Absoluta }\end{array}$} & \multicolumn{2}{|c|}{ Óbitos } & \multicolumn{2}{|c|}{ Total de Afetados } \\
\hline Tipo & Subtipo & & Abs & Média & Total & Média \\
\hline Terremoto & Movimento de terra & 1.003 & 1.066 .764 & $1.063,6$ & 181.401 .660 & $180.859,1$ \\
\hline Tempestades & Ciclone Tropical & 1.747 & 772.174 & 442,0 & 713.145 .455 & $408.211,5$ \\
\hline Seca & Seca & 621 & 700.644 & $1.128,3$ & 2.513 .998 .310 & $4.048 .306,5$ \\
\hline Terremoto & Tsunami & 36 & 251.968 & $6.999,1$ & 6.618 .951 & $183.859,8$ \\
\hline Temperaturas Extremas & Onda de Calor & 164 & 157.213 & 958,6 & 4.836 .879 & $29.493,2$ \\
\hline Epidemias & Doenças Bacterianas & 712 & 147.354 & 207,0 & 4.689 .263 & $6.586,0$ \\
\hline Inundação & Inundação Gradual & 2.561 & 123.268 & 48,1 & 2.514 .108 .010 & $981.690,0$ \\
\hline Inundação & Indeterminado & 1.212 & 105.793 & 87,3 & 873.293 .130 & $720.538,9$ \\
\hline Inundação & Enxurradas & 588 & 58.015 & 98,7 & 166.032 .203 & $282.367,7$ \\
\hline Epidemias & Doenças Virais & 437 & 53.683 & 122,8 & 7.003 .471 & $16.026,2$ \\
\hline Deslizamentos & Deslizamento & 519 & 32.000 & 61,7 & 9.895 .028 & $19.065,6$ \\
\hline Epidemias & Indeterminado & 138 & 27.032 & 195,9 & 2.988 .058 & $21.652,6$ \\
\hline Atividade Vulcânica & Queda de cinzas & 190 & 26.104 & 137,4 & 4.980 .188 & $26.211,5$ \\
\hline Tempestades & Indeterminado & 724 & 18.709 & 25,8 & 57.811 .678 & $79.850,4$ \\
\hline Temperaturas Extremas & Onda de Frio & 283 & 16.741 & 59,2 & 14.441 .111 & $51.028,7$ \\
\hline Tempestades & Tempestades Conectivas & 874 & 16.040 & 18,4 & 197.285 .952 & $225.727,6$ \\
\hline Epidemias & Doenças Parasitárias & 46 & 10.156 & 220,8 & 10.556 .528 & $229.489,7$ \\
\hline Outros Tipos & Outros Subtipos & 874 & 17.281 & 19,8 & 119.787 .814 & $137.057,0$ \\
\hline Total & & 12.729 & 3.600 .939 & 282,9 & 7.402.873.689 & $581.575,4$ \\
\hline
\end{tabular}

Fonte: EMDATA, 2017

As consequências desses eventos são sentidas de forma desigual entre as pessoas: a população menos favorecida, crianças e idosos são frequentemente os mais afetados em todo o planeta. Nesses termos o desenvolvimento de um arcabouço metodológico para estimativas e mensurações da vulnerabilidade sociodemográfica de indivíduos, populações e localidades aos diversos tipos de desastres é de fundamental importância.

Diversas instituições e pesquisadores têm proposto indicadores de risco e vulnerabilidade a desastres naturais. Os indicadores de abrangência internacionais têm a vantagem da comparabilidade entre países, mas, geralmente, são apresentados em uma escala pequena ou com abrangência de estados nacionais. Por outro lado, indicadores alternativos têm sido propostos para escalas maiores, com abrangência interurbana. Deve-se ainda ter em conta para a construção de indicadores de vulnerabilidade socioambiental é que o risco de morte, ferimentos ou perdas econômicas a um desastre é dependente do tipo de desastre que se analisa. Desastres muito localizados, como pequenas enchentes, tornados, deslizamentos, queda de raios e tremores de terra causam danos, feridos ou óbitos a um número limitados de indivíduos e destroem ou danificam um numero pequeno de estruturas. Por outro lado, grandes desastres naturais, a exemplo de furacões e ciclones tropicais, grandes terremotos, grandes erupções vulcânicas, tsunamis, grandes enchentes e secas prolongadas podem levar ao óbito centenas de milhares de pessoas e ferir um número bem maior, como também causar significantes perdas econômicas e até crises sociais em consequência direta ou indireta das perdas econômicas. Ou seja, cada desastre tem suas consequências baseado em conjunto correspondente de condições particulares de atividades e populações e, como também às especificidades de cada tipo de desastre. Dessa forma, entende-se que não é conceitualmente possível desenvolver um indicador que seja aplicável a todos os tipos de desastres. 
A proposta deste artigo é apresentar uma revisão sobre um conjunto de métodos e técnicas de mensuração da vulnerabilidade a desastres naturais, e propor um arcabouço preliminar que servirá de base para a construção de um indicador de Risco a Desastres Naturais, que seja aplicável (ou ajustável) a múltiplas escalas e coerente com as bases de dados disponíveis e com os desastres mais comuns no Brasil.

\section{Revisão Bibliográfica}

Antes de apresentar as metodologias revisadas, optou-se por fazer um breve estudo bibliométrico sobre desastres naturais para analisar a evolução da importância do mesmo no contexto científico internacional. Posteriormente foram elencadas técnicas e métodos de abrangência internacional e nacional propostos por diversas instituições. Embora esses indicadores sejam limitados às bases de dados utilizados para sua construção e abrangência territorial, as metodologias revisadas neste trabalho são um importante referencial teórico para a proposição de indicadores de risco e vulnerabilidade a desastres naturais.

\subsection{Estudo Bibliométrico}

A preocupação e a pesquisa sobre desastres naturais na literatura internacional são recentes. Uma busca no portal de periódicos CAPES/MEC, sem especificar uma base de publicações, utilizando a chave de busca "natural hazard", apresenta um total de 5.660 publicações, sendo 5.033 artigos, entre 1800 e 2017. Embora o intervalo de tempo apresentado no portal seja de 1800 a 2017, a primeira publicação é de 1937, Birth Injury in Relation to Labor, American Journal of Obstetrics and Gynecology, 1937, Vol.33(1), pp.1-18. Este artigo investiga as possíveis lesões ao feto causado por problemas relacionados ao trabalho da gestante. Localizou-se também uma publicação em 1938, The Journal of Pediatrics, 1938, Vol.13(6), pp.919-933. O artigo utiliza o termo natural hazard ao risco natural de infecção e testa o efeito de vacinas. A pesquisa no portal de periódicos apresenta uma publicação em 1900, relacionando o termo a mudança climática, contudo, a publicação associada é de uma tese de 2014, publicada em um repositório de teses australiano (TROVE) em 2015. Das duas ocorrências do período 1951-1960, uma tem origem na área médica e o outro na área de engenharia. Da mesma forma, das seis ocorrências da década seguinte cinco são associadas à medicina e um outro associado à biologia.

O volume de publicações aumenta de forma mais significativa a partir da década de 1970, quando são identificadas 60 publicações, a maior parte relacionada aos desastres ambientais (Gráfico 1). A partir desta década o volume cresce de forma consistente, o aumento mais expressivo a partir da década de 1990, se deve, possivelmente ao fato de a década ter sido designada como a International Decade for Natural Disaster Reduction (IDNDR) pela Union Nations Office for Disaster Risk Reduction (UNISDR). 


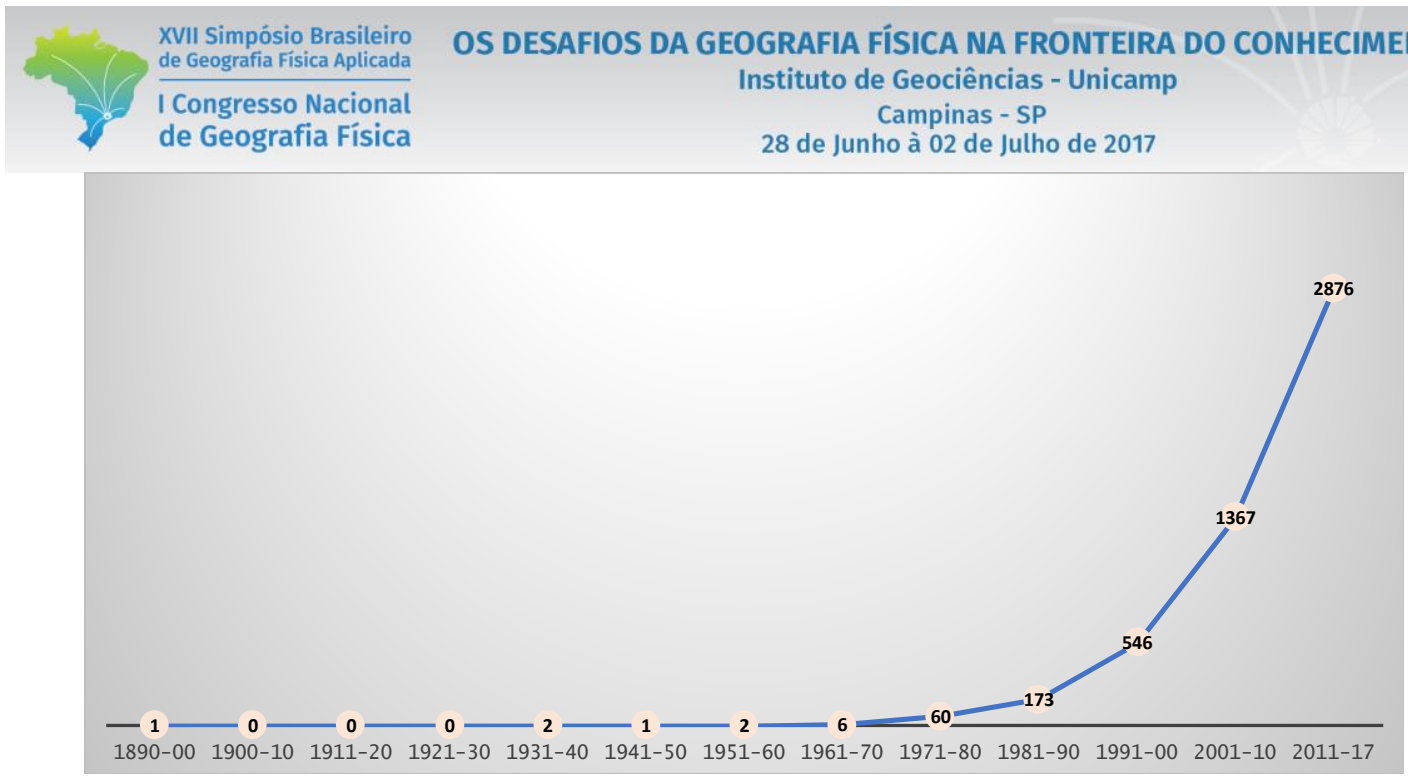

Gráfico 1

Publicações internacionais reportadas com a chave de busca "natural hazard”, por décadas - 1890-2017 Fonte: Portal de periódicos CAPES/MEC (2017).

Um detalhamento a partir de 1971, mostra que foi a partir de 2001 que as publicações que respondem a essa chave de busca cresceram de forma mais expressiva, conforme se verifica no gráfico 2.

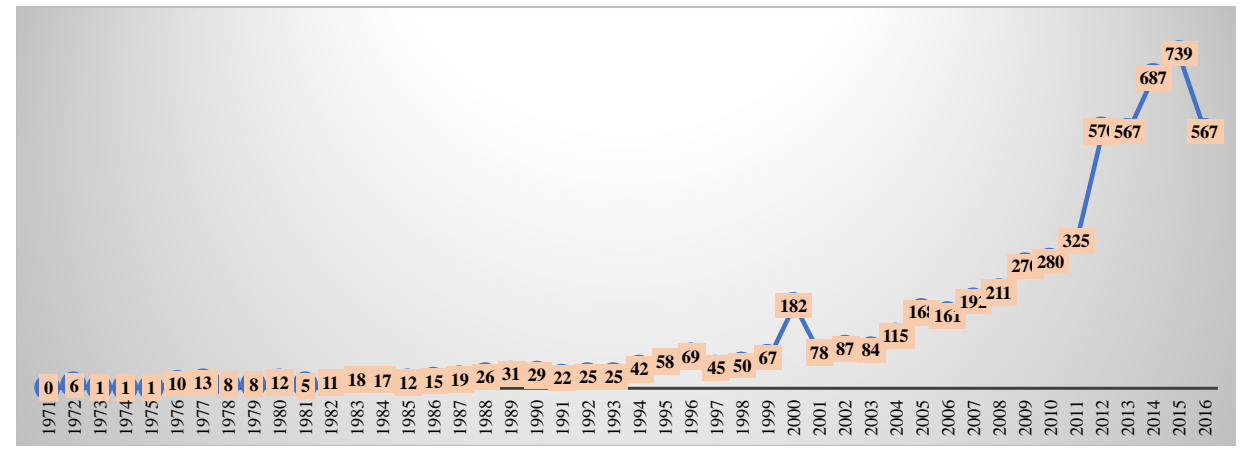

\section{Gráfico 2}

Publicações internacionais reportadas com a chave de busca “natural hazard", por ano - 1971-2016 Fonte: Portal de periódicos CAPES/MEC (2017).

A base de publicações que apresentou o maior volume foi a scopus (Elsevier) com 3.822 publicações no período. Contudo uma busca nessa base dados com a mesma chave de busca, reporta um número superior de publicações, em decorrência de algoritmos próprios. O Gráfico 3 mostra os artigos publicados por ano, entre 1962 e 2016, em resposta à chave de busca "natural hazard", como também com a chave de busca "social vulnerability" dentro dos resultados anteriores, com o intuito de identificar a presença dos dois termos nas mesmas publicações. A partir de 2007, aparecem um volume maior de publicações em que o termo social vulnerability aparece concomitantemente ao termo natural hazard. 


$\begin{aligned} & \text { XVII Simpósio Brasileiro } \\ & \text { de Geografia Fisica Aplicada }\end{aligned}$
$\begin{aligned} & \text { I Congresso Nacional } \\ & \text { de Geografia Física }\end{aligned}$

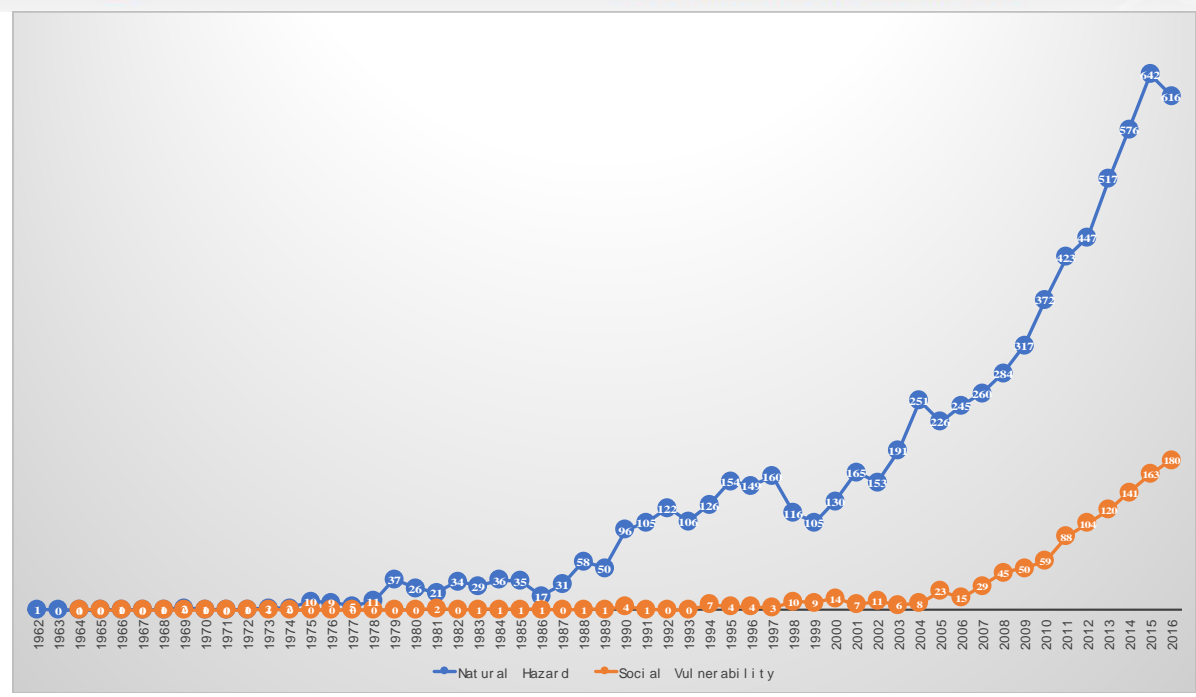

Gráfico 3

Publicações internacionais reportadas com a chave de busca "natural hazard" e "social vulnerability", por ano Fonte: Scopus (Elsevier) (2017). 1962-2016

Foi realizada uma pesquisa com chaves de busca em português no portal de periódicos CAPES/MEC com os termos comumente traduzidos, tais como desastre natural, risco de desastre, incluindo também a abordagem da vulnerabilidade social e ambiental, que é mais comum na literatura brasileira. Com a chave de busca "desastre natural" obtém-se 228 publicações sendo 24 em língua portuguesa. Com o termo "risco de desastre" obtém-se duas publicações, sendo uma em português. Já a busca com a expressão "vulnerabilidade ambiental" obtém-se 46 publicações, sendo $32 \mathrm{em}$ português. A associação da vulnerabilidade ambiental com a social, resulta em 64 publicações, sendo 47 em português, utilizando-se a expressão "vulnerabilidade socioambiental".

Essa breve análise da literatura internacional e nacional sobre desastres naturais mostra que a produção internacional é ampla e volumosa. O aumento do volume de publicações acompanha o início e o aumento da preocupação ambiental em termos globais marcada pela Conferência das Nações Unidas sobre o Meio Ambiente Humano (United Nations Conference on the Human Environment), realizada entre os dias 5 a 16 de junho de 1972 em Estocolmo. Essa foi a primeira reunião de chefes de estado organizada pelas Nações Unidas (ONU) focada nos problemas ambientais.

\subsection{Indicadores e abordagens internacionais}

A Disaster Risk Index - DRI, proposto pela United Nations Development Programme - UNDP, é apresentado como a primeira avalição dos fatores de risco a desastres por meio da comparação entre países da vulnerabilidade e exposição a três tipo de desastres naturais considerados como críticos: terremotos, ciclones tropicais e inundações (UNDP, 2004). Baseado na experiência da UNDP com o IDH o DRI pretendia ser o primeiro passo para a construção de um indicador capaz de mensurar o risco a desastres em nível global. A proposta do indicador seria fornecer dados e análises para apoiar tomadas de decisão nos níveis sub-nacionais, nacionais e internacionais. 


$\begin{aligned} & \text { XVII Simpósio Brasileiro } \\ & \text { de Geografia Fisica Aplicada }\end{aligned}$
$\begin{aligned} & \text { I Congresso Nacional } \\ & \text { de Geografia Física }\end{aligned}$$\quad \begin{gathered}28 \text { de Junho à } 02 \text { de Julho de } 2017 \\ \text { Campinas - SP }\end{gathered}$

O DRI permite o calculo do risco médio de morte para os três tipos de desastres naturais elencados, baseados em dados do EM-DAT de 1980 e 2000. O indicador também permite a identificar um conjunto de variáveis socioeconômicas e ambientais que são correlacionadas com o risco de morte. Na construção do DRI entende-se, intrinsicamente, que o risco ao desastre não é unicamente causado pelo desastre propriamente dito, mas é historicamente construído por meio de atividades e processos humanos. Ou seja, o risco de morte é apenas parcialmente dependente da presença de fenômeno físico e, no DRI, o risco refere-se exclusivamente ao risco de morte e exclui outras dimensões do risco, como as perdas econômicas e a própria subsistência de um grupo populacional após o desastre. O principal motivo dessa escolha é a ausência de dados confiáveis e disponíveis na escala global e com resolução nacional.

Outro ponto relevante à metodologia aplicada pelo DRI é que as pessoas, a infraestrutura e as atividades econômicas devem estar localizadas em uma área onde ocorrem um determinado desastre. Ou seja, necessariamente, deve haver uma exposição física e pessoas residindo em locais onde estes desastres ocorrem combinados com a frequência dos eventos. A exposição física não é um indicador de vulnerabilidade, mas é uma condição necessária para o risco de desastre existir, ou seja, a ausência de pessoas expostas indicaria, para o DRI, a inexistência de risco para a vida humana. A exposição física, entretanto, é insuficiente para explicar o nível de risco, pois, países com níveis semelhantes de exposição física a um determinado evento perigoso têm níveis de risco muito diferentes.

Para o DRI, o que define a magnitude de um risco é a exposição e a vulnerabilidade. Para o cálculo dos indicadores de vulnerabilidade,

(...) o DRI testa um conjunto de 26 variáveis sociais, econômicas e ambientais, selecionadas por um painel de especialistas, por meio de modelos de regressão múltipla logarítmica. As 26 variáveis selecionadas pertencem a oito categorias, a saber: econômica, tipo de atividade econômica, dependência e qualidade do ambiente, demográfica, saúde e saneamento, capacidade de alerta preventivo, educação, desenvolvimento. (BRAGA, et all., 2006, p.84)

As variáveis foram convertidas em médias para os 26 anos em análise e os resultados do modelo indicam parâmetros de maior ou menor risco.

O Global Hotspots Classification - GHC, proposto pelo Banco Mundial e Columbia University, avalia três componentes associados ao risco aos desastres naturais: (i) A probabilidade de ocorrência de diferentes tipos de desastres, (ii) os elementos expostos ao desastre, e (iii) a vulnerabilidade dos elementos expostos aos desastres. O arcabouço adotado para a definição de risco é comumente citado na bibliografia internacional sobre desastres naturais, segundo os autores do relatório. As análises consideram perdas econômicas e os impactos são analisados de duas formas: Single-hazard hotspots e Multi-hazard hotspots. A base de dados utilizada é o EM-DAT, em função do acesso público e da abrangência global (WBANK, 2005).

De forma distinta ao DRI, o GHC considera as perdas econômicas e o risco de morte, a seis tipos de desastres: ciclones, secas, enchentes, terremotos, erupções vulcânicas e deslizamentos de terra (WBANK, 2005). Devido às restrições da base de dados, os autores alertam que os resultados identificam áreas de alto 


$\begin{aligned} & \text { XVII Simpósio Brasileiro } \\ & \text { de Geografia Fisica Aplicada }\end{aligned}$
$\begin{aligned} & \text { I Congresso Nacional } \\ & \text { de Geografia Física }\end{aligned}$

risco relativo para desastres particulares, mas não são adequados para um diagnóstico de níveis absolutos de risco ou para comparações de padrões de risco entre diferentes tipos de desastres (BRAGA, et al., 2006). Um ponto importante da identificação dos hotsptos é o uso de uma base cartográfica internacional conhecida como Gridded Population of the World - GPW desenvolvida inicialmente pelo Center for International Earth Science Information Network - CIESIN. Esta base de dados transforma os dados censitários, de vários países em níveis subnacionais, em células regulares de "quadriláteros esféricos". A primeira versão do GPW considerava quadriláteros de 5' de longitude por 5' de latitude, que equivale a $85 \mathrm{~km}^{2}$ na linha do Equador. A segunda versão do GPW, utilizada na estimativa do GHC, considera quadriláteros de 2,5' de longitude por 2,5' de latitude e contém, para cada célula a população estimada para 1990, 1995 e 2000.

Utilizando a GPWv2, o projeto GHC estimou uma variedade de probabilidades de desastres e frequências extrapolando-as para uma base geoespacial comum. Foi também adicionado a cada célula da base geográfica, a densidade de estradas e ferrovias, de terra cultivada e valor agregado. Sendo assim, cada célula fornece estimativas da área total, da população, da densidade populacional, probabilidades a desastres e características associadas à exposição e vulnerabilidade. A células podem ser facilmente agregadas em diversos níveis territoriais e em diversas escalas.

O resultado final fornece uma medida relativa de risco convertido em um índice variando entre 1 e 10. Adotou-se como as áreas críticas (hotspots) aquelas células classificadas como 8, 9 ou 10, tanto para cada tipo de desastre quanto para a combinação deles que indicaria um risco múltiplo a desastres naturais.

\subsection{Indicadores e abordagens nacionais}

Maior e Candido (2014) apresentam uma revisão das metodologias brasileiras de vulnerabilidade socioambiental, associadas a problemática urbana.

(...) o quadro teórico, no qual se insere a vulnerabilidade socioambiental urbana, contempla a sobreposição (coexistência espacial) dos processos de expansão urbana envolvendo tanto a dispersão espacial de grupos de risco social, degradação ambiental e falta de serviços de infraestrutura urbana (MAIOR e CANDIDO, 2014).

Os autores observam que nos meios científicos brasileiros a vulnerabilidade socioambiental é tratada em nível local, identificando grupos populacionais submetidos a um alto risco em relação a desastres específicos. Maior e Candido (2014, p. 248) argumentam que os modelos metodológicos e teóricos apresentados por Alves (2005), Deschamps (2004; 2006), Almeida (2010), Hogan (2001) e Silveira (2010) partem de "um mesmo procedimento metodológico, baseado em dados do IBGE e da sobreposição cartográfica dos riscos ambientais com os riscos sociais distribuídos no espaço urbano estudado".

Alves (2005, 2010, 2013 e 2014) utiliza dados dos Censos Demográficos Brasileiros, por setor censitário, onde é identificado, incialmente, a vulnerabilidade social do setor com base na proporção de domicílios vulneráveis segundo variáveis socioeconômicas e do entorno, a exemplo, de renda, abastecimento de água, esgotamento sanitário, dentre outras. Em Alves (2010) a vulnerabilidade ambiental é identificada, por setor censitário, pela existência ou não de assentamentos precários, favelas, áreas urbanizadas e loteamentos 


$\begin{aligned} & \text { XVII Simpósio Brasileiro } \\ & \text { de Geografia Fisica Aplicada }\end{aligned}$
$\begin{aligned} & \text { I Congresso Nacional } \\ & \text { de Geografia Física }\end{aligned}$

irregulares. No demais trabalhos (Alves, 2005, 2013 e 2014) a vulnerabilidade ambiental é identificada de acordo a proporção da área do setor censitário que se localiza em áreas próximas de cursos d'água ou em áreas íngremes. A identificação qualitativa da vulnerabilidade social e ambiental como alta ou baixa produz uma tabela de contingência em que os setores são divididos em quatro categorias de vulnerabilidade socioambiental. Os estudos de Alves foram aplicados ao litoral paulista, regiões metropolitanas de São Paulo e Campinas, Cubatão, dentre outras áreas urbanas.

Almeida (2010) estuda as vulnerabilidades ambientais e sociais na região metropolitana de Fortaleza e estima um índice de vulnerabilidade socioambiental por meio da sobreposição de um índice de vulnerabilidade social com um índice de vulnerabilidade físico espacial a inundações. O indicador social faz uso de análise fatorial com um conjunto inicial de 59 variáveis do Censo Demográfico 2000 para cada setor censitário, identificando seis níveis de vulnerabilidade social. A vulnerabilidade ambiental, por sua vez, denominado pelo autor como Índice de Vulnerabilidade Físico-Espacial às Inundações - IVFI da bacia hidrográfica do rio Maranguapinho, propõe um "modelo espaciotemporal do risco de exposição às inundações mediante a extensão espacial dos eventos de inundação" (Almeida, 2010, p. 242), baseado na frequência dos eventos. O IVFI baseia-se na delimitação das áreas de inundação do rio Maranguapinho, de acordo com os referidos tempos de retorno das inundações e na utilização integrada de mapas, modelos digitais de terreno, simulações hidrológicas e hidráulicas. Sobrepondo-se os dados de vulnerabilidade social com os resultados do IVFI foram identificados seis níveis de vulnerabilidade socioambiental.

Hogan (2001), desenvolve um estudo de vulnerabilidade socioambiental no contexto urbano metropolitano de Campinas e aborda, os conceitos desenvolvidos por Katzman (1999) de capital físico, capital social e capital humano e classifica seus indicadores de acordo com esta nomenclatura. Para cada uma das dimensões foram realizadas procedimentos estatísticos e transformações matemáticas em que, quanto maiores os escores (mais próximo de um), piores são as condições relativas às dimensões. Os resultados são apresentados por cartografias que sobrepõe as áreas de vulnerabilidade social às de riscos ambientais.

Deschamps (2004 e 2006) apresenta um estudo extenso em que compara a vulnerabilidade socioambiental nas metrópoles brasileiras ${ }^{2}$. O estudo apresenta os procedimentos para a construção de tipologias que determinem espaços marcados por abrigar grupos socialmente vulneráveis. Para seu desenvolvimento são utilizados dados por setores censitários que foram agrupados segundo critérios de tamanho, em termos de domicílios e população; contiguidade; e homogeneidade, em relação a características populacionais e de infraestrutura. A metodologia trabalha com três dimensões (social, econômica e ambiental). A dimensão ambiental é mensurada pela ausência combinada de serviços básicos (esgotamento sanitário, abastecimento de água canalizada e coleta de lixo). A tipologia e o agrupamento das áreas nas regiões metropolitana fez São Paulo, Rio de Janeiro, Belo Horizonte, Porto Alegre, Brasília, Curitiba, Recife,
Fortaleza, Campinas, Manaus, Vitória, Goiânia, Belém, Florianópolis, Natal e Maringá 
uso de análise fatorial e análise de cluster e o resultado é apresentado na forma de uma leitura cruzada da vulnerabilidade social e risco ambiental.

\section{Discussão de métodos e escalas ideias.}

Para O'Riordan (2002), a vulnerabilidade a desastres é a incapacidade de uma pessoa, sociedade ou grupo populacional de evitar o perigo e os danos relacionados aos mesmos ou ao fato de ser forçado a viver em tais condições de perigo (Braga, et al., 2006). Cardona (2004), por sua vez, propõe analisar a vulnerabilidade a desastres por meio de componentes principais: fragilidade ou exposição; suscetibilidade; e falta de resiliência. Fragilidade, ou exposição, é o componente físico e ambiental, e é associado a estimativa do nível de exposição de um grupo a um desastre em função de sua localização e à ausência de resistência física à propagação do evento perigoso. Suscetibilidade é a componente socioeconômica e demográfica, associada à predisposição de uma população sofrer danos, decorrente do nível de marginalidade, segregação social e fragilidade econômica. A falta de resiliência é uma componente comportamental, comunitária e política, associada à capacidade de um grupo populacional absorver o choque de um desastre e se adaptar para voltar a um estado aceitável (BRAGA, et al., 2006).

É evidente que não é possível conceber um único indicador que seja capaz de estimar riscos e vulnerabilidades em níveis locais, regionais, nacionais ou globais. Assim como é notório que para cada tipo de desastre natural, existe um conjunto de variáveis sociais e econômicas que podem ser ou não associadas às consequências dos desastres.

Para o caso brasileiro, consideram-se como mais comuns, considerando número de pessoas afetadas na base de dados do EM-DAT, entre os anos de 1970 e 2016, os seguintes tipos de desastres naturais: Seca, com 78,8 milhões de pessoas; Enchentes, com 18,2 milhões de afetados; Epidemias, com 1,9 milhão. O pequeno registro de tempestades e deslizamentos pelo EM-DAT no Brasil vai de encontro com uma das limitações dessa base para estudos em escala subnacional, e citado no Trabalho do Banco Mundial e Columbia University (WBank, 2005): embora ampla e útil nas comparações internacionais, o EM-DAT desconsidera os níveis subnacionais e a apresenta uma pequena frequência de registros de eventos geofísicos ou hidrometeorológicos, seja porque ocorrem em locais isolados e pouco povoados ou porque não são registrados na imprensa internacional de língua francesa ou inglesa.

Adicionalmente, embora o EM-DAT registre um número expressivo de óbitos por epidemias no Brasil e no mundo, entende-se que a análise desse tema é complexa, pois depende da forma de proliferação de cada doença. Os óbitos por doenças infectocontagiosas são ainda eventos de registro compulsório na Organização Mundial da Saúde e com forte apelo para publicação na imprensa. Esses fatores combinados indicariam que as epidemias são mais impactantes para a população brasileira do que tempestades e deslizamentos, ponto que certamente deve ser melhor averiguado. 


\begin{tabular}{|c|c|}
\hline $\begin{array}{l}\text { XVII Simpósio Brasileiro } \\
\text { de Geografia Fisica Aplicada }\end{array}$ & $\begin{array}{l}\text { OS DESAFIOS DA GEOGRAFIA FISICA NA FRONTEIRA DO CONHECIMENTO } \\
\text { Instituto de Geociências - Unicamp }\end{array}$ \\
\hline $\begin{array}{l}\text { I Congresso Nacional } \\
\text { de Geografia Física }\end{array}$ & $\begin{array}{l}\text { Campinas - SP } \\
28 \text { de Junho à } 02 \text { de Julho de } 2017\end{array}$ \\
\hline
\end{tabular}

Tabela 2

Total de Desastres Naturais, Óbitos, Pessoas Afetadas e Perdas Econômicas, por Tipo de Desastre. Brasil. 19702016

\begin{tabular}{|c|c|c|c|c|c|c|c|}
\hline \multirow{2}{*}{$\begin{array}{l}\text { Desastre Natural } \\
\text { Tipo } \\
\end{array}$} & \multirow[t]{2}{*}{$\begin{array}{c}\text { Frequência } \\
\text { Absoluta }\end{array}$} & \multicolumn{2}{|c|}{ Óbitos } & \multicolumn{2}{|c|}{ Total de Afetados } & \multicolumn{2}{|c|}{$\begin{array}{l}\text { Perdas Econômicas } \\
\text { (Milhares US\$) }\end{array}$} \\
\hline & & Abs & Média & Total & Média & Total & Média \\
\hline Seca & 18 & 20 & 1,1 & 78.812 .000 & $4.378 .444,4$ & 11.183 .100 & $621.283,3$ \\
\hline Inundação & 116 & 5.542 & 47,8 & 18.175 .439 & $156.684,8$ & 9.214 .770 & $79.437,7$ \\
\hline Epidemias & 16 & 2.217 & 138,6 & 1.982 .376 & $123.898,5$ & 0 & 0,0 \\
\hline Tempestades & 16 & 159 & 9,9 & 226.390 & $14.149,4$ & 533.000 & $33.312,5$ \\
\hline Deslizamento & 20 & 878 & 43,9 & 91.000 & $4.550,0$ & 231.000 & $11.550,0$ \\
\hline Terremotos & 2 & 2 & 1,0 & 15.280 & $7.640,0$ & 5.000 & $2.500,0$ \\
\hline Incêndios & 3 & 1 & 0,3 & 12.000 & $4.000,0$ & 36.000 & $12.000,0$ \\
\hline Infestação de insetos & 1 & 0 & 0,0 & 2.000 & $2.000,0$ & 0 & 0,0 \\
\hline Temperaturas Extremas & 7 & 242 & 34,6 & 0 & 0,0 & 1.075 .000 & $153.571,4$ \\
\hline Total & 199 & 9.061 & 45,5 & 99.316.485 & $499.077,8$ & 22.277 .870 & $489.272,3$ \\
\hline
\end{tabular}

Fonte: EM-DAT, 2017

Nesses termos, considera-se como principais desastres naturais que deveriam ser contemplados em estudos subnacionais no Brasil: Secas, Enchentes, Tempestades e Deslizamentos.

As bases de dados a serem consideradas nesses estudos aplicados a realidade brasileira devem considerar a escala intra-municipal. Para a construção de variáveis confiáveis de risco manifesto, uma fonte de dados inestimável são os dados do AVADAN/SINDEC (documento oficial de comunicação de desastres do Sistema Nacional de Defesa Civil). Esta fonte traz dados sobre pequenos, médios e grandes eventos e é de abrangência municipal, mais adequado que as fontes internacionais. Os registros do AVANDAN trazem informações de mortalidade, pessoas afetadas, perdas econômicas, perdas de infraestrutura urbana e de infraestrutura de serviços de saúde e educação, dentre outros. O AVADAN foi adotado de forma obrigatória a partir de 2000, como parte integrante dos processos de declaração de estado de calamidade e emergência pública e é registrado nos três níveis federativos: município, unidades da federação e país. O registro nos três níveis federativos impacta na disponibilidade dos dados: municípios que tiveram seu decreto de calamidade decretado em nível municipal, podem não ter seu pedido homologado (registrado) em níveis estaduais ou federais. Outro ponto é que, tendo em vista o registro ser no nível municipal, raramente é identificado a localização geográfica do desastre na área do município. Dessa forma, para estudos do risco manifesto na escala intramunicipal dever-se-ia fazer uso de outras fontes de dados, a exemplo de registros na imprensa ou dos registros do Ministério da Saúde, como o Sistema de Informações de Mortalidade (SIM) e do Sistema de Internações Hospitalares (SIH) que identificam o endereço de residência da vítima, assim como a causa de morte ou internação. Para estimativa de indicadores da exposição ao risco, sugere-se o uso de ferramentas de geoprocessamento, sensoriamento remoto e modelos digitais de terreno.

Como base cartográfica, sugere-se avaliar o uso do GPWv4 do SEDAC. A versão 4 desta grade trabalha com células de 30" de latitude e longitude, o que equivale a aproximadamente a $1 \mathrm{~km}$ na linha do equador. Os dados originais foram interpolados e projetados para os anos de 2000, 2005, 2010, 2015 e 2020 . Os dados presentes no GPWv4 são população total, densidade populacional, área do solo, área da massa d'água e indicadores de qualidade dos dados (CIESIN, 2017). 


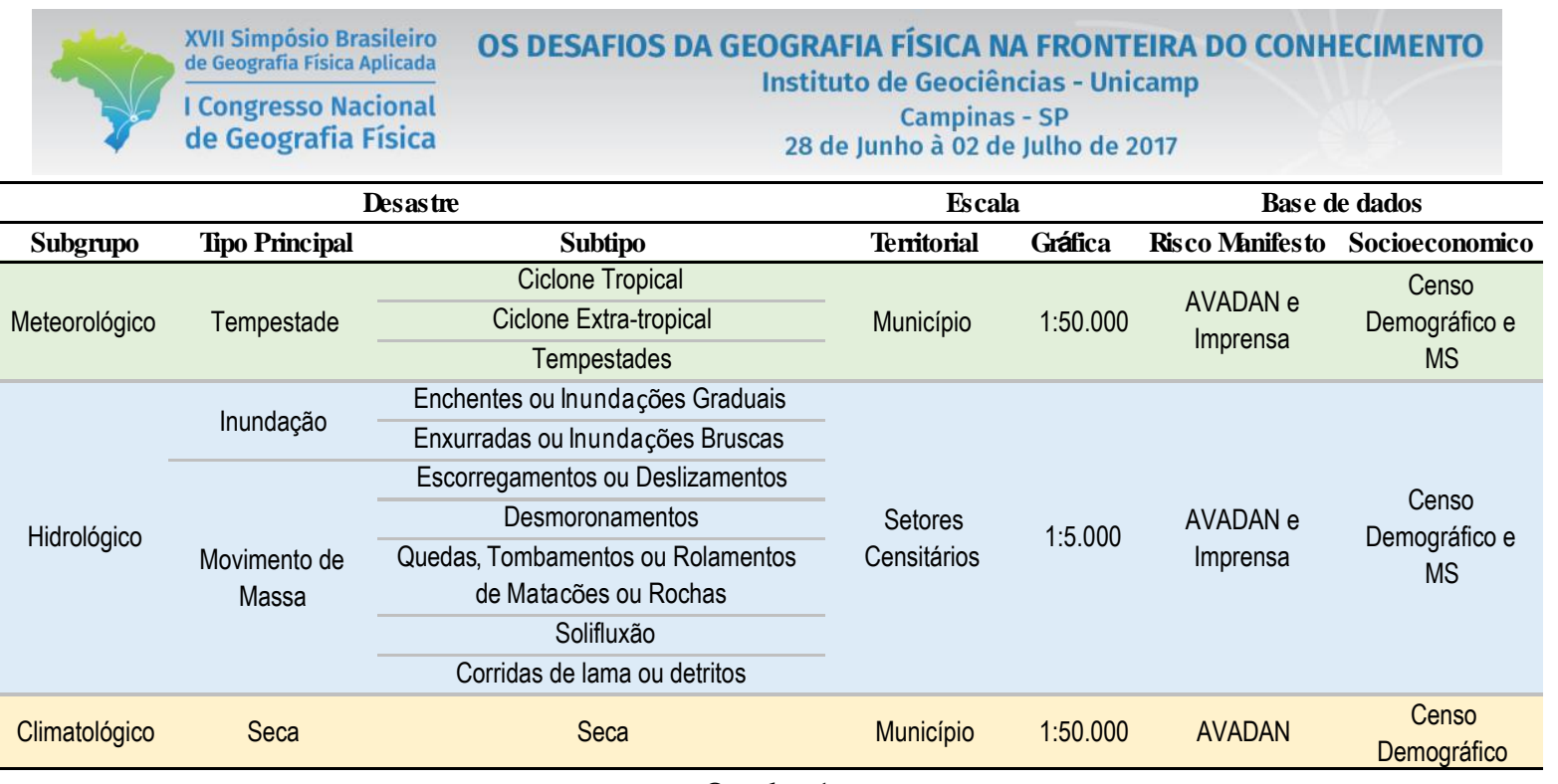

Quadro 1

Arcabouço preliminar para construção de indicadores de vulnerabilidade socioambiental a desastres naturais Fonte: Elaboração Própria

O quadro 1 sintetiza o arcabouço preliminar para a construção de indicadores de risco a desastres naturais, considerando os tipos de desastres elencados neste trabalho, a escala de análise, e as bases de dados a serem utilizadas. A proposta é trabalhar com os tipos principais de desastres, considerando que os desastres Hidrológicos devem ser analisados em escala intramunicipal, com destaque para as áreas urbanas, e que os desastres meteorológicos e climatológicos podem ser trabalhados na escala municipal ou até regional. As bases de dados serão adaptadas às escalas de análise e de acordo com a disponibilidade.

\section{Referencias bibliográficas}

ALMEIDA, L. Q. de. Vulnerabilidade socioambiental de rios urbanos: bacia hidrográfica do Rio Maranguapinho região metropolitana de Fortaleza-Ceará. Tese de doutorado. Rio Claro, Universidade Estadual Paulista "Júlio de Mesquita Filho". 2010.

ALMEIDA, Lutiane Queiroz de. Vulnerabilidades socioambientais de rios urbanos: bacia hidrográfica do rio Maranguapinho. Região metropolitana de Fortaleza, Ceará. 2010. 278 f. Tese (doutorado) - Universidade Estadual Paulista, Instituto de Geociências e Ciências Exatas, 2010. Disponível em: <http://hdl.handle.net/11449/104309>.

ALVES, H. P. et al. Dinâmicas de urbanização na hiperperiferia da metrópole de São Paulo: análise dos processos de expansão urbana e das situações de vulnerabilidade socioambiental em escala intraurbana. Revista Brasileira de Estudos Populacionais. Rio de Janeiro, v. 7, n. 1, pp. 141-159. 2010

ALVES, H. P. Vulnerabilidade sócio-ambiental na metrópole paulistana: uma análise das situações de sobreposição espacial de problemas e riscos sociais e ambientais. In: Anais. XI Encontro da Associação Nacional de Pós-graduação e Pesquisa em Planejamento Urbano e Regional (ANPUR), Salvador-BA. Salvador: ANPUR. 2005.

ALVES, Humberto Prates da Fonseca. Análise da vulnerabilidade socioambiental em Cubatão-SP por meio da integração de dados sociodemográficos e ambientais em escala intraurbana. Rev. bras. estud. popul., São Paulo, v. 30, n. 2, p. 349-366, Dec. 2013. Available from

<http://www.scielo.br/scielo.php?script=sci_arttext\&pid=S0102-30982013000200002\&lng=en\&nrm=iso>. access on 18 Mar. 2017. http://dx.doi.org/10.1590/S0102-30982013000200002

ALVES, Humberto Prates da Fonseca. Análise de um indicador de vulnerabilidade socioambiental da Região Metropolitana de Campinas no contexto das mudanças climáticas. In: Anais. XIX Encontro Nacional de Estudos Populacionais, ABEP, realizado em São Pedro/SP - Brasil. São Pedro: ABEP. 2014 


$\begin{gathered}\text { XVII Simpósio Brasileiro } \\ \text { de Geografia Fisica Aplicada }\end{gathered}$
$\begin{aligned} & \text { I Congresso Nacional } \\ & \text { de Geografia Física }\end{aligned}$

BRAGA, Tânia Moreira; OLIVEIRA, Elzira Lucia de; GIVISIEZ, Gustavo Henrique Naves. Avaliação de metodologias de mensuração de risco e vulnerabilidade social a desastres naturais associados à mudança climática. São Paulo perspect; 20(1): 81-95, jan.-mar. 2006. Disponível em <http://produtos.seade.gov.br/produtos/spp/v20n01/v20n01_06.pdf> Acessado em: 10-mar-2017

CARDONA O. D. et al. System of indicators for disaster risk management: main technical report. Manizales Washington: Instituto de Estudios Ambientales Universidad Nacional de Colombia / Inter-American Development Bank, 2005.

CIESIN. Center for International Earth Science Information Network. Columbia University. 2016. Gridded Population of the World, Version 4 (GPWv4): Administrative Unit Center Points with Population Estimates. Palisades, NY: NASA Socioeconomic Data and Applications Center (SEDAC). DIsponível em: <http://dx.doi.org/10.7927/H4F47M2C>. Acessado em: 10-mar-2017.

DESCHAMPS, M. V.. Vulnerabilidade socioambiental na região metropolitana de Curitiba. Tese de doutorado. Paraná, Universidade Federal do Paraná. 2004

DESCHAMPS, M. V.. Vulnerabilidade socioambiental nas regiões metropolitanas brasileiras. Brasília, Relatório de atividades do Observatório das Metrópoles. Convênio Ministério das Cidades/Observatório das Metrópoles/Fase/Ipardes. 2006

ELSEVIER [Base de dados de trabalhos científicos]. 2017. Disponível em: < https://www.elsevier.com.br>. Acesso em: 01 mar. 2017.

HOGAN, D. J. et al. (orgs). Migração e ambiente nas aglomerações urbanas. Campinas, Nepo/Unicamp. 2011

KAZTMAN, R. et al. Vulnerabilidad, activos y exclusión social en Argentina y Uruguay. Santiagodo Chile, OIT. (Documento de trabalho, pp. 107). 1999

MAIOR, Mônica Maria Souto; CANDIDO, Gesinaldo Ataíde. Avaliação das metodologias brasileiras de vulnerabilidade socioambiental como decorrência da problemática urbana no Brasil. Cad. Metrop., São Paulo, v. 16, n. 31, p. 241-264, June 2014. Available from

<http://www.scielo.br/scielo.php?script=sci_arttext\&pid=S2236-99962014000100241\&lng=en\&nrm=iso>. Acesso em 11 Mar. 2017. http://dx.doi.org/10.1590/2236-9996.2014-3111

O’RIORDAN, T. Precautionary Principle. In: Tolba , M.K. (Ed.). Encyclodia of Global Environmental Change. Chichester, UK: John Wiley, 2002. v. 4.

PERIODICOS CAPES [Base de busca de trabalhos científicos]. 2017. Disponível em:

<http://www.periodicos.capes.gov.br >. Acesso em: 01 mar. 2017.

SILVEIRA, H. (2010). Estudo da degradação e do impacto socioambiental na Bacia do Córrego Osório, Maringá - Paraná. Revista Geografar. Curitiba, v. 5, n. 1, pp. 176-205. Disponível em: http://www.ser.ufpr.br/geografar. Acesso em: 10 mar 2017.

UNDP. United Nations Development Programme. Reducing disaster risk: challenge for development. New Yory: UNDP, Bureau for Crisis Prevention and Recovery. 2004. Disponível em: < http://www.undp.org/content/undp/en/home/librarypage/crisis-prevention-and-recovery/reducing-disaster-risk-a-challenge-for-development.html > Acesso em 12/03/2016. ISBN 92-1-126160-0

WBANK, The World Bank. Natural Disaster Hotspots: A Global Risk Analysis. Disaster. Washington, D.C.: The World Bank, 2005. Risk Management Series, n.5. Disponível em: <http://documents. worldbank.org/curated/en/621711468175150317/pdf/344230PAPER0Na101officialOuse0only 1.pdf> Acesso em: 12/03/2016

\section{Agradecimentos}

À Fundação de Amparo à Pesquisa do Estado de São Paulo, projeto nº 2016/0007-3 pelo financiamento da pesquisa 